\title{
Increased Glutamate Levels in the Medial Prefrontal Cortex in Patients with Postpartum Depression
}

\author{
Alyssa M McEwen', Denee TA Burgess', Christopher C Hanstock ${ }^{2}$, Peter Seres ${ }^{2}$, Panteha Khalili', \\ Stephen C Newman', Glen B Baker', Nicholas D Mitchell', Janisse Khudabux-Der', Peter S Allen ${ }^{2}$ and \\ Jean-Michel LeMelledo*,I
}

'Department of Psychiatry, University of Alberta, Edmonton, AB, Canada; ${ }^{2}$ Department of Biomedical Engineering, University of Alberta, Edmonton, $A B$, Canada

\begin{abstract}
The medial prefrontal cortex (MPFC) is a key brain area in depressive symptomatology; specifically, glutamate (Glu) has been reported to play a significant role in major depression (MD) in this area. MPFC Glu levels are sensitive to ovarian hormone fluctuations and pregnancy and the postpartum period are associated with the most substantial physiological alterations of female hormones. It is therefore logical to measure MPFC Glu levels in women with postpartum depression (PPD). Using in vivo magnetic resonance spectroscopy (MRS) at a field strength of $3 \mathrm{~T}$, we acquired single-voxel spectra from the MPFC of 12 women with PPD and 12 healthy controls (HCs) matched for postpartum scan timing. Water-referenced MPFC Glu levels were measured using a MRS technique that allowed us to be specific for Glu with very little glutamine contamination. The concentrations of other water-quantified brain metabolites such as glycerophosphorylcholine plus phosphorylcholine, $N$-acetylaspartate (NAA), and creatine plus phosphocreatine were measured in the same MR spectra. MPFC Glu levels were higher in women with PPD $(7.21 \pm 1.20)$ compared to matched HCs $(6.04 \pm 1.21)$. There were no differences between groups for other brain metabolites measured. These findings suggest an association between Glu dysregulation in the MPFC and PPD. Whether the pathophysiology of PPD differs from the pathophysiology of MD remains to be determined. Further investigations are needed to determine the chronological associations between the occurrence of symptoms of PPD and the onset of changes in MPFC Glu levels.
\end{abstract}

Neuropsychopharmacology (2012) 37, 2428-2435; doi:I0.1038/npp.2012.101; published online 18 July 2012

Keywords: glutamate; postpartum; depression; magnetic resonance spectroscopy; medial prefrontal cortex; women

\section{INTRODUCTION}

Postpartum depression (PPD) is a common mood disorder that occurs in up to $20 \%$ of women after childbirth (Gaynes et al, 2005). According to the DSM-IV-TR (American Psychiatric Association, 2000), PPD is limited to a diagnosis of major depression (MD) with an onset within 4 weeks of birth. However, based on epidemiological data, it has been recommended by many researchers in the field that 3 months postpartum is a more suitable timeframe for defining postpartum onset of depression (Elliot, 2000; Wisner et al, 2006). Women who suffer from PPD experience feelings of inadequacy and hopelessness, which can often persist from months to years after childbirth. The damaging role that PPD plays in the mother-infant relationship may result in suboptimal cognitive and emotional development

*Correspondence: Dr J-M LeMelledo, Department of Psychiatry, Room IE7.|4, 8440 |12 Street Walter Mackenzie Center, University of Alberta, Edmonton, AB T6G 2B7, Canada, Tel: + I 7804076578 , Fax: + I 780407 6672, E-mail: jean-michel.lemelledo@ualberta.ca Received 9 January 20 I2; revised 24 April 20 I2; accepted 17 May 2012 in the child, which can subsequently increase the risk of depression in the child into adolescence (Ramchandani et al, 2005; Murray and Cooper, 2003; Goodman et al, 1993; Downey and Coyne, 1990; Rahman et al, 2004; Sinclair and Murray, 1998; Moehler et al, 2007; Murray et al, 2011). Although psychosocial aspects of PPD are well researched, there is a dearth of biological investigations into the pathophysiology of this disorder. As a result, it is not clear whether the pathophysiology of PPD differs from the pathophysiology of MD.

In vivo magnetic resonance spectroscopy (MRS) is the only noninvasive technique that can directly assess levels of neurochemicals, such as the excitatory neurotransmitter glutamate (Glu), in localized brain regions (Stanley, 2002; Soares et al, 1996). The ability to measure Glu selectively by MRS is confounded by overlapping resonances with glutamine (Gln) owing to a similarity in the chemical structures of the two.

MRS investigations have suggested dysregulation of glutamix (a combination of Glu and Gln) in patients with MD (Yuksel and Ongur, 2010). Although the glutamatergic hypothesis of depressive symptomatology has been prominent 
for a period of time, the recent clinical findings of the rapid onset of antidepressant activity of the N-methyl-D-aspartate (NMDA) antagonist ketamine (Berman et al, 2000; Zarate et al, 2006) have established the glutamatergic hypothesis of depression as one of the most promising avenues of research.

Various neuroimaging investigations have suggested that the medial prefrontal cortex (MPFC) is a key brain area for depressive symptomatology (Price and Drevets, 2010). Recent animal studies (Li et al, 2010, 2011; Autry et al, 2011) suggest that the rapid antidepressant response to ketamine is related to its synaptogenesis activity in the MPFC. Furthermore, data indicates that MPFC Glu levels are influenced by ovarian hormone fluctuations (Batra $\mathrm{et} \mathrm{al}$, 2008). Of note, pregnancy and the postpartum period are associated with the most substantial physiological alterations of female hormones (Buckwalter et al, 1999; Bloch et al, 2003). As PPD is defined as an episode of MD occurring during the postpartum phase, it stands to reason that glutamatergic dysregulation in the MPFC may play a key role in depressive symptomatology observed in PPD.

Our objective in this report was to examine whether Glu levels in the MPFC are decreased or increased in women with PPD compared to healthy women.

\section{MATERIALS AND METHODS}

\section{Subjects}

In all, 12 women suffering from PPD (1 suffering from PPD as defined by the DSM-IV-TR, presenting with symptoms within the first 4 weeks postpartum and the other 11 suffering from PPD with onset of symptoms in the more commonly accepted time frame of within the first 3 months postpartum) were matched with 12 healthy control women (HCs) based on postpartum scan time. Women were brought in for the MRS scan as soon as possible after first contact was made. One PPD woman and her matched HC were scanned at 3 weeks postpartum, two PPD women and their matched controls were scanned at 5 weeks postpartum, one PPD woman and her matched HC were scanned at 7 weeks postpartum, two PPD women and their matched HCs were scanned at 9 weeks postpartum, and six PPD women and their matched HCs were scanned at 3 months postpartum. All participants were recruited from advertisements and through collaborations with health institutions in Edmonton, Canada. All subjects were compensated for their time. Each woman was recruited according to the guidelines of the Health Research Ethics Board of the University of Alberta. After a complete description of the study was provided to the subjects, written informed consent was obtained.

Eligible women were not taking any psychotropic drugs or herbal products with psychotropic activity 3 months before entering the study or at any time during the study. Participants were excluded if they had used any street or recreational drugs in the previous 6 months or during the study, or if they used any form of hormonal contraception. A pregnancy test ensured that women were not pregnant. Other factors that excluded participants included potential confounding factors such as brain injury or classical contraindications to magnetic resonance imaging.
All participants were administered the Structured Clinical Interview for DSM-IV-TR of Axis I disorders to screen for any current or lifetime Axis I psychiatric disorders. Specific exclusion criteria for HCs included any current or past Axis I psychiatric illness. PPD women had to meet the diagnostic criteria for MD with an onset of symptoms within the first 3 months postpartum. The Edinburgh Postpartum Depression Scale (EPDS) and the Beck Depression Inventory (BDI) were administered to all participants in order to screen for PPD. MRS sessions for both PPD patients and HCs were scheduled during the follicular phase of the menstrual cycle (with the exception of women who were scanned early in the postpartum at a time when menstruation has not yet resumed).

\section{MRS}

MRS was performed in the Peter $S$ Allen MR Research Centre, University of Alberta, Edmonton, Canada, using a stimulated echo acquisition mode (STEAM) sequence (Frahm et al, 1989) and a $3 \mathrm{~T}$ magnet (Magnex Scientific, Concord, CA) equipped with a spectrometer (Surrey Medical Imaging System, Surrey, UK) and a quadrature birdcage resonator. A $2 \times 3 \times 3 \mathrm{~cm}^{3}$ voxel (for segmentation and spectroscopy) was positioned such that the $2 \mathrm{~cm}$ dimension was perpendicular to, and centered on, the midline. The center sagittal slice was subsequently used to first register the voxel such that the posterior edge touched the rostrum of the corpus callosum in the mid-sagittal plane and inferior edge lay along the anterior commissureposterior commissure (AC-PC) line. The voxel was then rotated until the corners of the anterior edge were equidistant from the brain surface, while maintaining one corner contacting the AC-PC line, and an edge contacting the corpus callosum (Figure 1).

Shimming to $<0.05$ p.p.m. was accomplished by using both FASTMAP (Gruetter, 1993) and an in-house autoshim routine. The optimal in vivo Glu and Gln contrast to

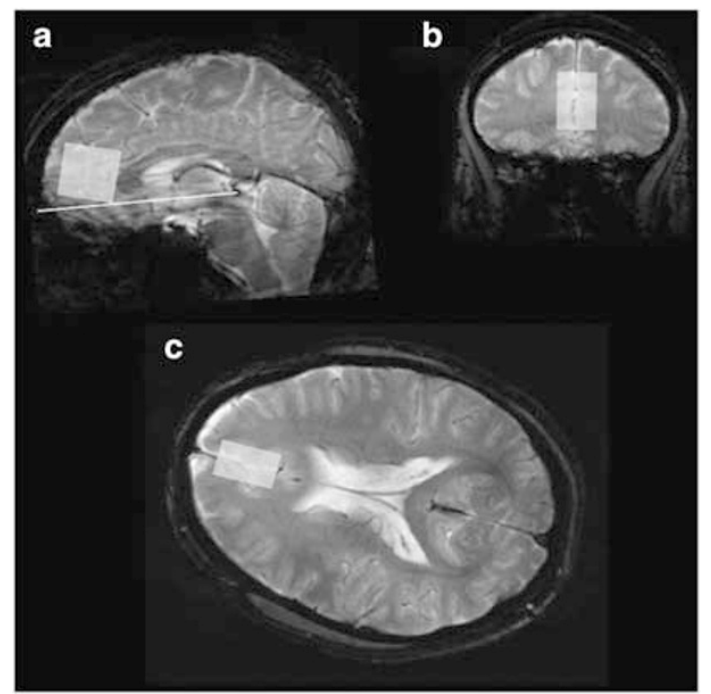

Figure I Medial prefrontal cortex voxel shown in (a) mid-sagittal section with the posterior inferior corner contacting the anterior commissureposterior commissure line, (b) coronal, and (c) transverse views. 
background, determined using numerical simulation, used a TE equal to $240 \mathrm{~ms}$, mixing time (TM) equal to $27 \mathrm{~ms}$, and repetition time (TR) equal to $3 \mathrm{~s}$ (Thompson and Allen, 2001). The long TE time resulted in minimal macromolecule contamination due to their short $T_{2}$ relaxation time (Behar et al, 1994). Spectra were the sum of 512 averages, acquired in 16 blocks of 32 averages. This warranted each of the 16 subspectra to be analyzed for spectral artifacts because of subject movement or hardware fluctuations before their final summing (Zhu et al, 1992). Where necessary, reregistering of each of the 16 subspectra to the same frequency reference before summing was allowed. The in vivo data were analyzed using the LCModel (version 6.0-1) analysis program (Provencher, 1993). The metabolite basis spectra used in the LCModel analysis were derived by numerical simulation and included $N$-acetylaspartate (NAA), creatine plus phosphocreatine ( $\mathrm{t}-\mathrm{Cr}$ ), myo-inositol, $\mathrm{N}$-acetylaspartylglutamate, taurine, lactate, aspartate, glycine, alanine, $\gamma$-aminobutyric acid, glycerophosphorylcholine plus phosphorylcholine (t-Cho), and Glu. This analysis gave reliable measures of Glu, NAA, $\mathrm{t}-\mathrm{Cho}$, and $\mathrm{t}-\mathrm{Cr}$ in the MPFC, with Cramer-Rao Lower Bound of the fit for Glu $<13 \pm 4 \%$, for NAA $<3 \pm 1 \%$, for $\mathrm{t}-\mathrm{Cr}<9 \pm 3 \%$, and for $\mathrm{t}-\mathrm{Cho}<5 \pm 1 \%$ in PPD patients.

We only report for Glu, NAA, t-Cr, and $t$-Cho. Glu measures from LCModel analysis typically had a standard deviation of the fit $<20 \%$ and were therefore deemed reliable. Selection of the target Glu signal at 2.35 p.p.m. was optimized using numerical simulation by minimizing contamination from overlapping signals (Figure 2), and by assessing the TE and TM space of the simulated response of Glu, Gln, NAA, GABA, glutathione (GSH), and homocarnosine spin systems to find the optimal Glu signal-tobackground (TE, TM $=240,27 \mathrm{~ms}$ ). The reduced $\mathrm{SNR}$ at this longer TE required us to select a relatively large $2 \times 3 \times 3 \mathrm{~cm}^{3}$ volume, and signal average for $\sim 26 \mathrm{~min}(512$ averages, $\mathrm{TR}=3 \mathrm{~s}$ ). Moreover, placement across the midline to maximize the Glu-rich GM was critical to yield a good Glu signal. Under these optimal timing conditions, contamination from other metabolites was Gln $8 \%$, NAA $11 \%$, and GABA 7\%. Signals with minimal contamination include GSH and homocarnosine. A representative spectrum used for LCModel analysis is shown in Figure 3.

Segmentation of the frontal brain region was performed using a double-inversion recovery $1-\mathrm{D}$ projection method (Hanstock and Allen, 2000). The segmentation data were employed to scale the water data used for quantification. The water content has been well characterized in both gray matter (GM) $80 \%$, and white matter (WM) $65 \%$. In addition, these data allowed us to eliminate the cerebrospinal fluid (CSF) water volume that contributes to the total water signal, so that the quantified metabolite concentrations relate to the tissue space of the GM and WM. All computations necessary for calculating experimental timings before acquisition and for data analysis were performed using the MATLAB program environment.

The water data for quantification were acquired at several TE values $(\mathrm{TE}=20,40,60,80,100,150,200,250,300,350$, 400, 450, 500, 700, 900, 110, 1300, $1500 \mathrm{~ms}$; TR = $12000 \mathrm{~ms}$; 2 averages per TE value). Using customized processing routines in MATLAB, the water data were imported, and filtered, Fourier-transformed, and phase and baseline
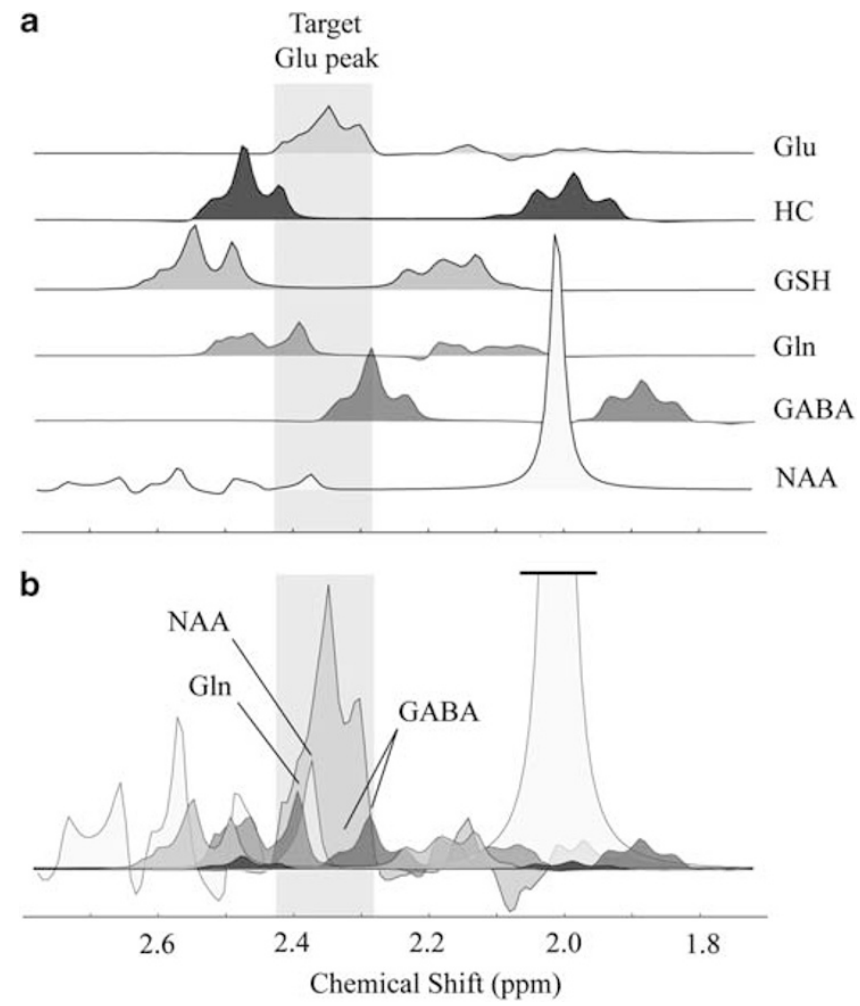

Figure 2 Simulation used to optimize the stimulated echo acquisition mode (STEAM) settings. (a) Simulated metabolite magnetic resonance (MR) spectra for STEAM $\{$ TE,TM $\}=\{240,27 \mathrm{~ms}\}$ centered on the spectral region surrounding the Glu multiplet at $\sim 2.35$ p.p.m. Metabolites at equimolar concentrations include: glutamate (Glu), glutamine (Gln), glutathione (GSH), homocarnosine (HC), $\gamma$-aminobutyric acid (GABA), and $N$-acetylaspartate (NAA). (b) Scaled metabolite spectra based on typical literature concentrations (relative to Glu at 100\%), NAA (I20\%), GABA (I 5\%), HC (3\%), GSH (20\%), and Gln (40\%). No significant overlap with the target Glu signal arises from GSH or HC. Since the NAA-asparate signal amplitude is well characterized by its singlet, its overlap as a contaminating signal can be readily accounted for during LCModel analysis. Within the Glu target band, only Gln (Gln peak/Glu peak 8\%) and GABA ( $\sim 7 \%$ ) contamination will have a minor impact on quantification under these optimized stimulated echo acquisition mode (STEAM) acquisition timings.

corrected. The water peak area from each spectrum in this TE series was determined and these area data were fitted to a multi-exponential using a non-negative least-squares algorithm. This fitting yielded both the $T_{2}$ relaxation time coefficients contributing to the decay and their relative proportions. In addition, it permitted an estimation of the water peak area at a theoretical TE of $0 \mathrm{~ms}$.

\section{Metabolite quantification}

Three series of data were used for quantification, which were acquired from the same selected voxel: Metabolite peak area estimates are extracted from the LCModel output; segmentation information for GM, WM, and CSF compartment sizes is used to estimate water concentration in the selected brain voxel, and internal water data acquired at different TE values are used as the reference MR signal standard. 


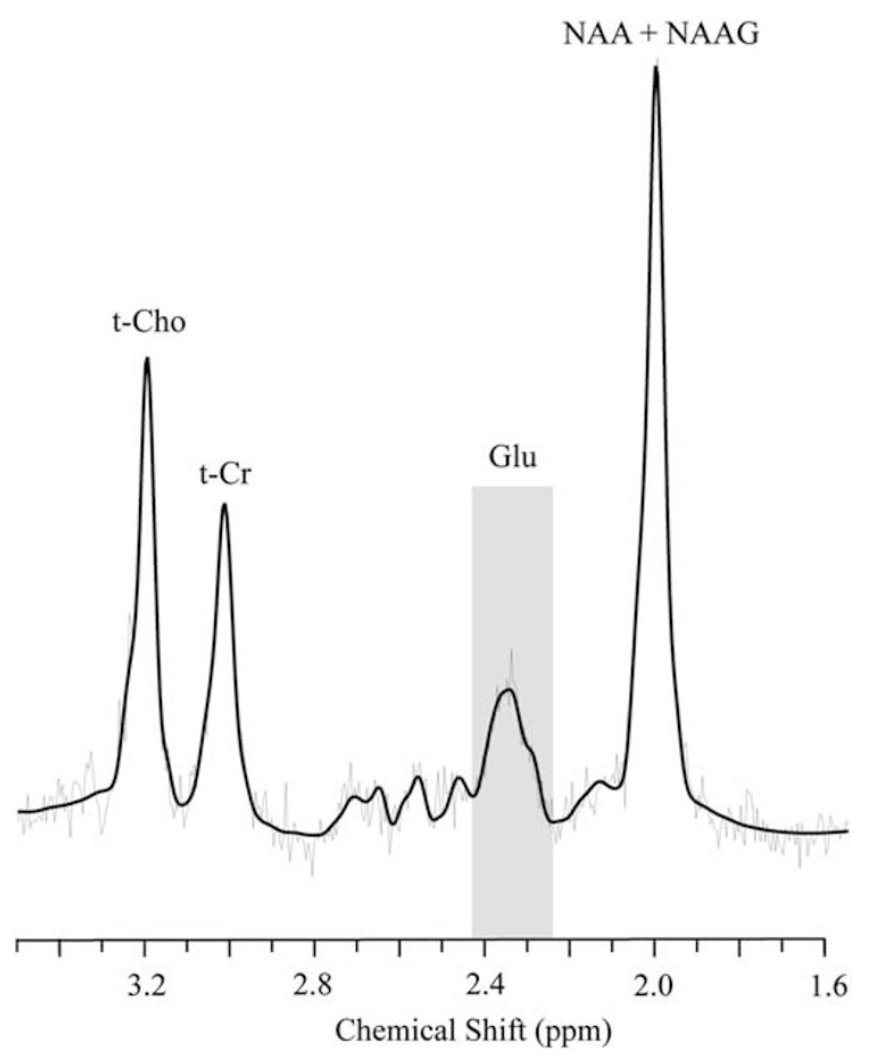

Figure 3 Sample stimulated echo acquisition mode (STEAM) localized magnetic resonance spectroscopy (MRS) data acquired from the medial prefrontal cortex and with sequence timings optimized for recovering signal from glutamate (STEAM TE, TM=240, $27 \mathrm{~ms}$ ). The spectra illustrate the unfiltered data superimposed with the LCModel fit in red. Glu, glutamate; $\mathrm{NAA}+\mathrm{NAAG}, \mathrm{N}$-acetylaspartate plus $\mathrm{N}$-acetylaspartylglutamate; $\mathrm{t}$ - $\mathrm{Cr}$, creatine plus phosphocreatine; t-Cho, glycerophosphorylcholine plus phosphorylcholine; p.p.m., parts per million.

Metabolite and water MR signals ( $S_{\text {metab }}$ and $S_{\text {water }}$, respectively) and concentrations ( $C_{\text {metab }}$ and $C_{\text {water }}$, respectively) are related by the simple expression:

$$
S_{\text {water }} / C_{\text {water }}=S_{\text {metab }} / C_{\text {metab }}
$$

After rearranging the simple expression, the metabolite concentration can be readily calculated:

$$
C_{\text {metab }}=\left(C_{\text {water }} \times S_{\text {metab }}\right) / S_{\text {water }}
$$

The term $C_{\text {water }}$ is calculated by accounting for the GM:WM compartments:

$$
\begin{aligned}
C_{\text {water }}= & \text { Pure Water Conc } \times\left[\left(\mathrm{GM}_{\text {segment }} \times \mathrm{GM}_{\text {water }}\right)\right. \\
& \left.+\left(\mathrm{WM}_{\text {segment }} \times \mathrm{WM}_{\text {water }}\right)\right]
\end{aligned}
$$

where

$$
\begin{aligned}
& \mathrm{GM}_{\text {water }}=0.8 \times \text { Pure Water Conc } \\
& \mathrm{WM}_{\text {water }}=0.65 \times \text { Pure Water Conc } \\
& \text { Pure Water Conc }=1000 / \mathrm{MW}_{\text {water }}=55.56 \mathrm{M}
\end{aligned}
$$

The term $S_{\text {water }}$ is calculated from NNLS fitting of the water data acquired at multiple TE values and extrapolation of the brain water compartment to a theoretical $\mathrm{TE}=0 \mathrm{~ms}$.
The term $S_{\text {metab }}$ can be used directly as measured $\left(S_{\text {metabTE240 }}\right)$, or can be scaled to account for the effects of metabolite $T_{2}$ and the different number of averages used for water-suppressed and non-suppressed acquisitions.

$$
S_{\text {metab }}=S_{\text {metabTE240 }} \times \mathrm{SF}_{T_{2} \text { metab }} \times \mathrm{SF}_{\mathrm{av}}
$$

where for data acquired at $\mathrm{TE}=240 \mathrm{~ms}$, and using 512 or 2 averages (water-suppressed or non-suppressed), scaling factors for the difference in the number of averages used $\left(\mathrm{SF}_{\mathrm{av}}\right)$, and for the metabolite $\mathrm{T}_{2} \quad\left(\mathrm{SF}_{T_{2} \text { metab }}\right)$ can be accounted for by the following:

$$
\begin{aligned}
& \mathrm{SF}_{\mathrm{av}}=\text { sqrt } 2 / \text { sqrt } 512 \\
& \mathrm{SF}_{T_{2} \text { metab }}=\exp \left(-240 / T_{2 \text { metab }}\right)
\end{aligned}
$$

Note that $T_{2}$ values for metabolites were assigned based on averaged literature values for NAA (350 ms), Cr (150 ms), and Cho $(310 \mathrm{~ms})$, and estimated for Glu $(380 \mathrm{~ms})$ based on expected normal brain concentration values for the GM:WM mix sampled in our studies. Also note that the scaling factor accounting for metabolite $T_{2}$ is only to provide numerical values in the $\mathrm{mM}$ range, and that the same values are applied to all data. This allows comparison to reported data. One could equally well report the data in institutional units by using the measured metabolite signal $S_{\text {metabTE240 }}$.

\section{Analysis}

A two-tailed $t$-test was used for independent sample analysis of variables between $\mathrm{HCs}$ and PPD women. Additional covariate analysis was also performed treating $\% \mathrm{GM}$ as a covariate. Statistical significance was defined to be $p \leqslant 0.05$. An analysis of the relationship between the EPDS and BDI scores and neurochemical concentrations was conducted using the Pearson correlation coefficient.

\section{RESULTS}

The mean age of the women with PPD was $28.67 \pm 7.45$ years and the mean age of the HCs was $29.08 \pm 4.89$ years $(t=0.43$, d.f. $=11, p=0.68)$. None of the PPD women had current co-morbid psychiatric disorders, but 5 out of the 12 PPD women had a history of MD. MPFC Glu levels were significantly increased in PPD women compared to matched HCs (see Table 1). In all cases, MPFC Glu levels were higher in the PPD woman compared to her matched HC. There were no statistically significant differences between PPD women and matched HCs for the other MPFC metabolite levels (NAA, t-Cr, and t-Cho) or tissue composition (GM, WM, and CSF) (Table 1). Although there was no statistically significant difference between groups in \%GM content in the MPFC voxel, a lower $p$-value $(p=0.10)$ lead us to perform a covariate analysis treating $\% \mathrm{GM}$ as a covariate. When the covariate analysis was performed, the increase in Glu levels in PPD women remained marginally significant $(p=0.067)$. No significant differences were observed upon covariate analysis for the other metabolites, including NAA that is also mainly present in GM. 
Table I Water-Referenced Metabolite Concentrations and Tissue Composition in Women with PPD and Healthy Subjects

\begin{tabular}{|c|c|c|c|c|c|c|}
\hline & \multicolumn{2}{|c|}{$\begin{array}{l}\text { PPD patients } \\
\quad(n=12)\end{array}$} & \multicolumn{2}{|c|}{$\begin{array}{l}\text { Control } \\
\text { subjects } \\
(n=12)\end{array}$} & \multicolumn{2}{|c|}{ Group } \\
\hline & Mean & SD & Mean & SD & $\begin{array}{c}p- \\
\text { value }\end{array}$ & $\stackrel{t}{\text { (d.f. }=I I)}$ \\
\hline \multicolumn{7}{|c|}{ Metabolite } \\
\hline Glu & 7.21 & 1.20 & 6.04 & 1.21 & $0.02^{\mathrm{a}}$ & 2.77 \\
\hline NAA & 9.89 & 1.07 & 9.10 & 1.28 & 0.19 & 1.41 \\
\hline $\mathrm{t}-\mathrm{Cr}$ & | 1.87 & 3.23 & 10.22 & 2.57 & 0.11 & 1.72 \\
\hline $\mathrm{t}-\mathrm{Cho}$ & 1.97 & 0.28 & 1.84 & 0.30 & 0.25 & 1.20 \\
\hline$\% G M$ & 57.88 & 8.22 & 50.03 & 13.47 & 0.10 & 1.83 \\
\hline$\% W M$ & 27.29 & 6.58 & 29.73 & 10.66 & 0.62 & 0.52 \\
\hline$\% C S F$ & $\mid 4.83$ & 11.67 & 20.25 & 9.48 & 0.27 & 1.17 \\
\hline
\end{tabular}

Abbreviations: CSF, cerebrospinal fluid; $H C s$, healthy controls; Glu, glutamate; GM, gray matter; NAA, N-acetylaspartate; PPD, postpartum depression; t-Cho, glycerophosphorylcholine plus phosphorylcholine; t-Cr, creatine plus phosphocreatine; WM, white matter.

Brain metabolites are measured in institutional units.

${ }^{a} \mathrm{~A}$ significant difference indicated between groups.

The Pearson correlation coefficient was used to assess the association between depressive symptoms (based on scores from the BDI or EPDS) and water-referenced neurochemicals, including Glu, in PPD women. There was no statistically significant correlation between water-referenced neurochemicals, including Glu, and scores on either EPDS or BDI in PPD women. BDI and EPDS scores were not available for two PPD women. Results of the analysis between EPDS and BDI scores with Glu were $r=-0.281 \quad(p=0.43)$ and $r=-0.165 \quad(p=0.65)$, respectively; t-Cho results were $r=-0.078(p=0.83)$ and $r=0.0 .099(p=0.79)$, respectively; NAA results were $r=-0.106(p=0.77)$ and $r=-0.037$ $(p=0.92)$, respectively; and $\mathrm{t}-\mathrm{Cr}$ results were $r=-0.002$ $(p=0.99)$ and $r=-0.074(p=0.84)$, respectively.

\section{DISCUSSION}

This study suggests that Glu levels in the MPFC are increased in PPD women compared to HCs. To the best of our knowledge, no other study has investigated brain Glu levels in PPD. However, MRS investigations have been performed in patients with MD. Most MRS investigations of the MPFC in MD have measured Glx $(\mathrm{Glu}+\mathrm{Gln})$ and found decreased levels of Glx in the MPFC of patients suffering from MD. A MRS investigation performed by Hasler et al (2007) found that 20 unmedicated MD patients demonstrated a decrease in Glx concentrations in the MPFC when compared to controls. Two other studies in the anterior cingulate cortex (ACC) support findings of decreased Glx in MD patients compared to HCs (Auer et al, 2000; Pfleiderer et al, 2003). However, without Glu and Gln levels being individually measured, it is difficult to discuss these findings in relation to our results. Clear resolution of the Glu signal from Gln is not considered feasible at lower field strengths such as $1.5 \mathrm{~T}$. By increasing the field strength, this ability is improved (Tkac et al, 2001); however, although it has been shown that shorter echo times (TE) can produce a reliable distinction of the Glu peak from Gln (Mullins et al, 2008), the best timing conditions need to have the PRESS sequence echo timings set to be asymmetric (Jutras et al, 2009). In addition, the use of an optimized STEAM sequence with a longer $\mathrm{TE}$ in our study enabled us to measure the Glu signal with minimal contamination from the Gln signal or macromolecules.

A study by Merkl et al (2011) used a field strength of $3 \mathrm{~T}$ ( $\mathrm{TE}=80 \mathrm{~ms}$ ) to measure water-quantified Glu concentrations, with little contamination from the Gln signal, in the prefrontal cortex of MD patients. Merkl et al (2011) found Glu to be reduced in the ACC in MD patients compared to HCs. This study suggests that Glu levels in the MPFC are decreased in MD, which is opposite to our results of increased MPFC Glu levels in PPD women. In contrast to results provided by Merkl et al, in a post-mortem study, Hashimoto et al (2007) found elevated Glu levels in the frontal cortex of MD patient brains compared to $\mathrm{HC}$ brains.

Both male and female participants were used in the previously mentioned studies, and although closely matched for age (Auer et al, 2000) and age and sex (Hasler et al, 2007; Pfleiderer et al, 2003; Merkl et al, 2011), the phase of the menstrual cycle was not taken into consideration for female participants who were not menopausal, and may have altered the results. Indeed, our research team has shown that Glu levels are decreased during the luteal phase compared to the follicular phase of the menstrual cycle (Batra et al, 2008).

Pregnancy and the postpartum period are associated with fluctuations in female hormones (ie, estrogen and progesterone) and associated neuroactive steroids (NASs) that have been hypothesized to be a contributing factor to the pathophysiology of PPD. Levels of estrogen, progesterone and NASs (such as pregnenolone and allopregnanolone) rise during pregnancy and return to pre-pregnancy levels in the postpartum (Bloch et al, 2003; Gilbert Evans et al, 2005). Bloch et al (2000) induced symptoms of depression in euthymic women with a history of PPD, but not in HCs, by exposing women to 8 weeks of hormonal treatment with both estrogen and progesterone, followed by an immediate withdrawal period meant to mimic pregnancy and the postpartum.

The link between Glu and female hormones in relation to depression in humans is poorly understood at this time. Our research group has demonstrated that MPFC Glu levels fluctuate in relation to the rise and fall of female hormones and associated NASs during the menstrual cycle (Batra et al, 2008). NASs as well as estrogen and progesterone are able to cross the blood-brain barrier, acting as neuromodulators by binding to neurotransmitter receptors and altering neuronal excitability (Finocchi and Ferrari, 2011; Rupprecht, 2003). In animal studies, estrogen has been shown to increase the number of NMDA receptors on dendritic spines by means of increased mRNA production, as well as to increase NMDA-mediated Glu receptor activity (Woolley and McEwen, 1993; Smith, 1989). Progesterone, on the other hand, has been shown to have inhibitory effects, reducing the number of dendritic spines and excitatory synapses 
(Woolley and McEwen, 1993). Animal studies have also shown both that NASs can modulate glutamatergic neurons (Zamudio-Bulcock and Valenzuela, 2011) and that Glu impacts the production of NASs (Remage-Healey et al, 2008). Considering the known interactions between female hormones, NASs and glutamatergic activity, it is therefore possible that the fluctuations of female hormones and NASs in the postpartum contribute to the increased MPFC Glu fluctuations observed in PPD women. It is important to note that our current findings apply to the follicular phase of the menstrual cycle and not the luteal phase (for those women who had resumed menses).

Only one MRS study has previously been performed in PPD women. That pilot study, which consisted of nine PPD women, found that GABA levels in the occipital cortex did not differ between PPD women and HCs scanned within 6 months postpartum and who had not yet resumed menstruation (Epperson et al, 2006). Since Glu is the major excitatory neurotransmitter in the cortex and its action is counterbalanced by the inhibitory action of GABA, this study is remotely relevant to our investigation. However, considering the region-specific alterations of brain metabolites in MD (Hasler et al, 2007), an absence of GABA level dysregulation in the MPFC of PPD women cannot be inferred from the data obtained from the occipital cortex.

We acknowledge the contingency that the increased MPFC Glu levels in PPD women may be correlated to past psychiatric disorders of these women and more particularly to MD rather than to the current PPD. However, this contingency is not supported by previous MRS studies which have suggested that alterations in Glx levels associated with MD resolve to levels that are similar to that of controls following clinical treatment (Yildiz-Yesiloglu and Ankerst, 2006). Furthermore, Taylor et al (2009) compared levels of Glx in the ACC of unmedicated individuals with a history of MD to those of controls and found no significant differences between groups. Consistent with these findings, there were no differences in Glu levels in the five PPD women with a history of MD (7.17 \pm 1.47$)$ relative to the seven PPD women without a history of MD $(7.25 \pm 1.01)(t=-0.11$, d.f. $=10, p=0.92)$.

We did not find a correlation between the severity of depressive symptoms and MPFC Glu levels, and this is consistent with the results of other MRS investigations performed in MD patients (Hasler et al, 2007; Sanacora et al, 1999). Indeed, in those investigations, which demonstrated dysregulations of Glu levels in various brain regions of MD patients, no correlations were observed between the severity scores of various depression scales and the Glu levels (Sanacora et al, 2004). However, a greater sample size would have allowed for a more subtle analysis of the relationship between Glu levels and the severity of PPD symptoms, as has been done in this study.

As priority was given to matching for postpartum scan time, women were not matched for breastfeeding. Coincidentally when breastfeeding was taken into consideration, there were only 3 out of 12 pairs that were unmatched for breastfeeding. Breast feeding status is therefore an unlikely confounding factor.

The relatively small sample size is a limitation to our study, and replication with a larger sample size would allow for further analysis into the underlying pathophysiology of PPD. Although Glu concentration was our main variable, the MRS methodology chosen allowed for concomitant measurements of other metabolites and we reported on these. As a result of these multiple comparisons, a type I error cannot be excluded; however, we do not have the sample size to allow for Bonferroni corrections. Furthermore, our sample is a mix of PPD women with early or late onset PPD, which has been defined as being either before or after 6 to 8 weeks from delivery, respectively (Dennis, 2004). Indeed, it has been suggested that the pathophysiology of early onset and late onset PPD may differ (Dennis, 2004). The heterogeneity of our PPD population is a commonly occurring issue in published PPD research (Stowe et al, 2005). In most published PPD studies, women with either a pregnancy onset, with an early onset, or with a late onset are included and analyzed as a whole.

Referencing Glu to water has a significant advantage (compared to referencing to other metabolites) for the interpretation of MRS data. Indeed, we can ensure that the Glu level fluctuations that we observed are not related to the fluctuation of another brain metabolite used as an internal reference. While an advantage of MRS is the measurement of total tissue Glu, it does not differentiate intracellular and extracellular glutamatergic activity (Valentine and Sanacora, 2009). Therefore, the precise involvement of the mechanism accountable for Glu dysregulation remains unclear. ${ }^{13} \mathrm{C}$-MRS technology has the potential to be used for the measurement of the precise involvement of the glutamatergic system by combining intravenous infusion of ${ }^{13} \mathrm{C}$-labeled glucose, or other precursors, with MRS (Valentine and Sanacora, 2009); this type of investigation would enable us to improve our overall understanding of the observed changes in Glu levels.

In conclusion, this is the first report investigating brain Glu levels in PPD women. Our findings of increased MPFC Glu levels have to be tempered by the fact that the difference between MPFC Glu levels in PPD women and HCs was only marginally statistically significant in the covariate analysis for GM content. Our results, however, contrast with findings of lower MPFC Glu levels in MD patients. Replication of our findings is necessary and future MRS investigations with sample sizes sufficient to control for PPD onset (early $v s$ late), and including women suffering from $\mathrm{MD}$ as an additional control group will help us refine our understanding of the role of Glu in the pathophysiology of PPD.

\section{ACKNOWLEDGEMENTS}

We thank the Misericordia Unit 3E and the Grey Nuns anteand postpartum unit for their support. We would also like to thank the University of Alberta Hospital Foundation. This research was sponsored by a Canadian Institutes of Health Research (CIHR) grant to Dr Jean-Michel Le Melledo, Dr Peter Allen, Dr Glen Baker, Dr Chris Hanstock, and Dr Steve Newman, and by CIHR and a Wyeth Ayerst Research and Development Grant for a post-doctoral fellowship to Dr Panteha Khalili. JMLM received salary support from the Alberta Heritage Foundation for Medical Research (AHFMR) during this investigation. 


\section{DISCLOSURE}

The authors report no competing interests. Over the past 3 years, GBB has had compensation from the following, although these funds are unrelated to the current manuscript: The CIHR (operating grants); Pfizer Pharmaceuticals (an educational grant and an operating grant), the manufacturer of ziprasidone and pregabalin; and SinoVeda Canada (operating grant), a company involved with natural products.

\section{REFERENCES}

American Psychiatric Association (2000). Diagnostic and Statistical Manual of Mental Disorders 4th edn. Text Revision. American Psychiatric Association: Washington, DC.

Auer PD, Putz B, Kraft E, Lipinski B, Schill J, Holsboer F (2000). Reduced glutamate in the anterior cingulate cortex in depression: an in vivo proton magnetic resonance spectroscopy study. Biol Psychiatry 47: 305-313.

Autry A, Adachi M, Nosyreva E, Na E, Los M, Cheng P et al (2011). NMDA receptor blockade at rest triggers rapid behavioural antidepressant responses. Nature 475: 91-97.

Batra NA, Seres-Mailo J, Hanstock C, Seres P, Khudabux J, Bellavance $\mathrm{F}$ et al (2008). ${ }^{1} \mathrm{H}$ MRS measurement of brain glutamate levels in premenstrual dysphoric disorder. Biol Psychiatry 63: 1178-1184.

Behar KL, Rothman DL, Spencer DD, Petroff OA (1994). Analysis of macromolecule resonances in ${ }^{1} \mathrm{H}$ NMR spectra of human brain. Magnet Reson Med 32: 294-302.

Berman RM, Cappiello A, Anand A, Oren DA, Heninger GR, Charney DS et al (2000). Antidepressant effects of ketamine in depressed patients. Biol Psychiatry 47: 351-354.

Bloch M, Daly RC, Rubinow DR (2003). Endocrine factors in the etiology of postpartum depression. Compr Psychiatry 44: 234-246.

Bloch M, Schmidt PJ, Danaceau M, Murphy J, Nieman L, Rubinow DR (2000). Effects of gonadal steroids in women with a history of postpartum depression. Am J Psychiatry 157: 924-930.

Buckwalter JG, Stanczyk FZ, McCleary CA, Bluestein BW, Buckwalter DK, Rankin KP et al (1999). Pregnancy, the postpartum, and steroid hormones: effects on cognition and mood. Psychoneuroendocrinology 24: 69-84.

Dennis CL (2004). Can we identify mothers at risk for postpartum depression in the immediate postpartum period using the Edinburgh Postnatal Depression scale? J Affect Disord 78: 163-169.

Downey G, Coyne JC (1990). Children of depressed parents: an integrative review. Psychol Bull 108: 50-76.

Elliott SA (2000). Report on the Satra Bruk workshop on classification of postnatal mental disorders. Arch Womens Ment Health 3: 27-33.

Epperson CN, Gueorguieva R, Czarkowski KA, Stiklus S, Sellers E, Krystal JH et al (2006). Preliminary evidence of reduced occipital GABA concentrations in puerperal women: a ${ }^{1} \mathrm{H}-\mathrm{MRS}$ study. Psychopharmacology 186: 425-433.

Finocchi C, Ferrari M (2011). Female reproductive steroids and neuronal excitability. Neurological Sci 32(Suppl 1): S31-S35.

Frahm J, Bruhn H, Gyngell ML (1989). Localized high resolution proton NMR spectroscopy using stimulated echoes: initial applications to human brain in vivo. Magnet Reson Med 9: 79.

Gaynes BN, Gavin N, Meltzer-Brody S, Lohr KN, Swinson T, Gartlehner G et al (2005). Perinatal depression: prevalence, screening accuracy, and screening outcomes. Evidence Rep/ Technol Assess (Summary) 119: 1-8.
Gilbert Evans S, Ross L, Sellers E, Purdy R, Romach M (2005). $3 \alpha$ Reduced neuractive steroids and their precursors during pregnancy and the postpartum period. Gynecol Endocrinol 21: 268-279.

Goodman SH, Brogan D, Lynch ME, Fielding B (1993). Social and emotional competence in children of depressed mothers. Child Dev 64: 516-531.

Gruetter R (1993). Automatic, localized in vivo adjustment of all first- and second-order shim coils. Magnet Reson Med 29: 804-811.

Hanstock CC, Allen PS (2000). Segmentation of brain from a PRESS localized single volume using double inversion recovery for simultaneous T1 nulling. In: Eighth Annual Meeting of the International Society for Magnetic Resonance in Medicine. Denver, USA.

Hashimoto K, Sawa A, Iyo M (2007). Increased levels of glutamate in brains from patients with mood disorders. Biol Psychiatry 62: 1310-1316.

Hasler G, van de Veen JW, Tumonis T, Meyers N, Shen J, Drevets WC (2007). Reduced prefrontal glutamate/glutamine and gamma-aminobutyric acid levels in major depression determined using proton magnetic resonance spectroscopy. Arch Gen Psychiatry 64: 193-200.

Jutras JD, Hanstock CC, Snyder J, Wilman AH (2009). PRESS spectroscopy of glutamate: effects of voxel location and field strength. Proceedings of the 17th International Society for Magnetic Resonance in Medicine, Honolulu.

Li N, Lee B, Liu RJ, Banasr M, Dwyer J, Iwata M et al (2010). mTOR-dependent synapse formation underlies the rapid antidepressant effects of NMDA antagonists. Science 329: 959-964.

Li N, Liu RJ, Dwyer J, Banasr M, Lee B, Son H et al (2011). Glutamate $N$-methyl-D-aspartate receptor antagonists rapidly reverse behavioral and synaptic deficits caused by chronic stress exposure. Biol Psychiatry 69: 754-761.

Merkl A, Schubert F, Quante A, Luborzewski A, Brakemeier EL, Grimm S et al (2011). Abnormal cingulate and prefrontal cortical neurochemistry in major depression after electroconvulsive therapy. Biol Psychiatry 69: 772-779.

Moehler E, Kagan J, Parzer P, Brunner R, Reck C, Wiebel A et al (2007). Childhood behavioral inhibition and maternal symptoms of depression. Psychopathology 40: 446-452.

Mullins PG, Chen H, Xu J, Caprihan A, Gasparovic C (2008). Comparative reliability of proton spectroscopy techniques designed to improve detection of J-coupled metabolites. Magnet Reson Med 60: 964-969.

Murray L, Arteche A, Fearon P, Halligan S, Goodyer I, Cooper P (2011). Maternal postnatal depression and the development of depression in offspring up to 16 years of age. J Am Acad Child Adolesc Psychiatry 50: 460-470.

Murray L, Cooper P (2003). Intergenerational transmission of affective and cognitive processes associated with depression: infancy and the preschool years. In: Goodyer I (ed). Unipolar Depression: A Lifespan Perspective. Oxford University Press: Oxford.

Pfleiderer B, Michael N, Erfurth A, Ohrmann P, Hohmann U, Wolgast $M$ et al (2003). Effective electroconvulsive therapy reverses glutamate/glutamine deficit in the left anterior cingulum of unipolar depressed patients. Psychiatry Res 122: 185-192.

Price JL, Drevets WC (2010). Neurocircuitry of mood disorders. Neuropsychopharmacology 35: 192-216.

Provencher SW (1993). Estimation of metabolite concentrations from localized in vivo NMR spectra. Magnet Reson Med 30: 672-679.

Rahman A, Ibqual Z, Bunn J, Lovel H, Harrington R (2004). Impact of maternal depression on infant nutritional status and illness: a cohort study. Arch Gen Psychiatry 61: 946-952. 
Ramchandani P, Stein A, Evans J, O’Connor TG, ALSPAC study team (2005). Paternal depression in the postnatal period and child development: a prospective population study. Lancet 365: 2201-2205.

Remage-Healey L, Maidment NT, Schlinger BA (2008). Forebrain steroid levels rapidly fluctuate during social interactions. Nat Neurosci 11: 1327-1334.

Rupprecht R (2003). Neuroactive steroids: mechanism of action and neuropsychopharmacological properties. Psychoneuroendicrinology 28: 139-168.

Sanacora G, Gueorguieva R, Epperson CN, Wu YT, Appel M, Rothman DL et al (2004). Subtype-specific alterations of gammaaminobutyric acid and glutamate in patients with major depression. Arch Gen Psychiatry 61: 705-713.

Sanacora G, Mason GF, Rothman DL, Behar KL, Hyder F, Petroff OA et al (1999). Reduced cortical gamma-aminobutyric acid levels in depressed patients determined by proton magnetic resonance spectroscopy. Arch Gen Psychiatry 56: 1043-1047.

Sinclair D, Murray L (1998). Effects of postnatal depression on children's adjustment to school. Br J Psychiatry 172: 58-62.

Smith SS (1989). Estrogen administration increases neuronal responses to excitatory amino acids as a long term effect. Brain Res 503: 354-357.

Soares JC, Krishnan KR, Keshavan MS (1996). Nuclear magnetic resonance spectroscopy: new insights into the pathophysiology of mood disorders. Depression 4: 14-30.

Stanley JA (2002). In vivo magnetic resonance spectroscopy and its application to neuropsychiatric disorders. Can J Psychiatry 47: 315-326.

Stowe ZN, Hostette AL, Newport DJ (2005). The onset of postpartum depression: implications for clinical screening in obstetrical and primary care. Am J Obstetr Gynecol 192: $522-526$.
Taylor MJ, Selvaraj S, Norbury R, Jezzard P, Cowen PJ (2009). Normal glutamate but elevated myo-inositol in anterior cingulate cortex in recovered depressed patients. J Affect Disord 119: 186-189.

Thompson RB, Allen PS (2001). Response of metabolites with coupled spins to the STEAM sequence. Magnet Reson Med 45: 955-965.

Tkac I, Anderson P, Adriany G, Merkle H, Ugurbil K, Gruetter R (2001). In vivo ${ }^{1} \mathrm{H}$ NMR spectroscopy of the human brain at $7 \mathrm{~T}$. Magnet Reson Med 46: 451-456.

Valentine GW, Sanacora G (2009). Targeting glial physiology and glutamate cycling in the treatment of depression. Biochem Pharmacol 78: 431-439.

Wisner KL, Chambers C, Sit DKY (2006). Postpartum depression: a major public health problem. J Am Med Assoc 21: 2615-2618.

Woolley CS, McEwen BS (1993). Roles of estradiol and progesterone in regulation of hippocampal dendritic spine density during the estrous cycle in the rat. J Comp Neurol 336: 293-306.

Yildiz-Yesiloglu A, Ankerst DP (2006). Review of ${ }^{1} \mathrm{H}$ magnetic resonance spectroscopy findings in major depressive disorder: a meta-analysis. Psychiatry Res 147: 1-25.

Yuksel C, Ongur D (2010). Magnetic resonance spectroscopy studies of glutamate-related abnormalities in mood disorders. Biol Psychiatry 68: 785-794.

Zamudio-Bulcock PA, Valenzuela CF (2011). Pregnenolone sulfate increases glutamate release at neonatal climbing fibre to Purkinje cell synapses. Neuroscience 175: 24-36.

Zarate Jr CA, Singh JB, Carlson PJ, Brutsche NE, Ameli R, Luckenbaugh DA et al (2006). A randomized trial of an $N$ methyl-D-aspartate antagonist in treatment-resistant major depression. Arch Gen Psychiatry 63: 856-864.

Zhu G, Gheorghiu D, Allen PS (1992). Motional degradation of metabolite signal strengths when using STEAM: a correction method. NMR Biomed 5: 209-211. 